\title{
Neon-FIB for the Fabrication of Tips for Atom Probe Tomography and Electron Tomography
}

Frances Allen ${ }^{1}$, John Notte ${ }^{2}$, Deying Xia ${ }^{2}$, Paul Blanchard ${ }^{3}$, Ruopeng Zhang ${ }^{1}$, Andrew Minor ${ }^{4}$ and Norman Sanford ${ }^{3}$

${ }^{1}$ UC Berkeley, Berkeley, California, United States, ${ }^{2}$ Carl Zeiss, Peabody, Massachusetts, United States, ${ }^{3}$ NIST, Boulder, Colorado, United States, ${ }^{4}$ UC Berkeley and LBNL, Berkeley, California, United States

Focused ion beam (FIB) milling is routinely used for the site-selective removal of material on the microscale down to the nanoscale. Applications of FIB milling include cross-sectioning for imaging of internal structures by Scanning Electron Microscopy (SEM), thinning of specimens to electron transparency for Transmission Electron Microscopy (TEM), and the fabrication of specific geometries such as needles to form samples for Atom Probe Tomography (APT). The most widely used type of FIB is the gallium FIB, based on the liquid metal ion source. However, for certain applications the effects of gallium contamination can be a major limiting factor. Over the last decade several new FIB sources have been introduced offering a wider choice of ion species and beam parameters [1]. The source producing the smallest spot size for applications requiring the highest resolution is the atomically sharp Gas Field Ionization Source (GFIS), first developed as the Helium Ion Microscope and then extended to operate with neon [2]. Neon FIB takes advantage of the greater mass of neon versus helium to enable sputtering at a faster rate, while retaining the resolution advantage of a beam formed from ionization events at a single atom - as well as the advantage that the neon beam is chemically inert [3].

For APT, samples must be needle-shaped with a tip diameter of $<100 \mathrm{~nm}$. This geometry produces a high local electric field at the apex of the biased sample and thus enables field-evaporation of ions. Importantly, the needles must also be very robust and not fracture under the electrostatic stress imposed by the applied electric field. Needle-shaped specimens are also implemented in TEM tomography, preferably with even sharper tips. Here, the needle geometry enables acquisition of image projections over all tilt angles eliminating the so-called 'missing wedge' of information from an inaccessible angular range due to the geometric constraints of a planar sample. In gallium FIB, gallium ions become implanted near the surface and can diffuse along grain boundaries and interfaces. Implanted gallium may even react chemically with the specimen, and lead to liquid metal embrittlement [4]. Thus, for materials particularly affected by gallium contamination (e.g. aluminum and its alloys, group III-V semiconductors, and various thin-film structures) we put forward an alternative approach in which gallium FIB performs the initial milling, but neon FIB performs the final milling steps to produce ultrasharp gallium-free needle specimens. This builds on previous work in which a similar gallium/neon sequential approach was used to produce gallium-free lamellar samples for TEM [5]. We propose that the neon FIB technique will allow APT and TEM tomography of a range of materials and material systems, which are currently hampered by conventional, gallium-only, FIB preparation methods.

References

[1] N. S. Smith, J. A. Notte, and A. V. Steele, MRS Bulletin 39 (2014) 329-335

[2] R. H. Livengood et al., NIMA 645 (2011) 136-140

[3] F. I. Allen et al., Nanoscale 11 (2019) 1403-1409

[4] K. A. Unocic, M. J. Mills, and G. S. Daehn, J. Microscopy 240 (2010) 227-238

[5] T. C. Pekin, F. I. Allen, and A. M. Minor, J. Microscopy 264 (2016) 59-63 